\title{
In-Vitro Effect of Natural Honey on the Growth of Streptococcus sanguinis
}

\author{
A Alaee ${ }^{1}$, A Azizi ${ }^{2}, N_{\text {Valaei }}^{3}$, S Taheri ${ }^{4}$, F Ravanshadi ${ }^{5}$, \\ M Hajipour ${ }^{6}$, M Fattahi $^{\text {T }}$
}

1-Assistant Professor, Oral medicine Dept, Member of Dental Material Research Center,Dental Faculty, Islamic Azad University of Medical Sciences, Tehran, Iran.

2- Professor,Oral Medicine Dept,Dental Faculty, Islamic Azad University of Medical Sciences, Tehran, Iran.

3- BSC, Faculty member of Thalasemia Research center, Mazandaran ,Iran.

4- Associate Professor Dept microbiology Shahid Beheshti University, Tehran,Iran.

5- Dentist, Tehran, Iran.

6-Dentist, Vancouver, BC, Canada.

7- Post Grduated Students, Oral Medicine Dept, Faculty of Dentistry, Tehran Medical Sciences, Islamic Azad University, Tehran, Iran

\begin{tabular}{l}
\hline ARTICLE INFO \\
\hline Article History \\
Received: Nov 2018 \\
Accepted: Dec 2018 \\
ePublished: Jan 2019 \\
\hline Corresponding author: \\
M Fattahi, Post Gr- \\
duated Students, Oral \\
Medicine Dept, Faculty \\
of Dentistry, Tehran \\
Medical Sciences, Is- \\
lamic Azad University, \\
Tehran, Iran \\
Email: Maryam.fat- \\
tahi2010@gmail.com
\end{tabular}

ABSTRACT

Background and Aim: Streptococcus sanguinis (S. sanguinis) is one of the effective bacteria in the formation of caries and periodontal diseases which are caused by dental plaque. Currently, the use of natural honey $(\mathrm{NH})$ is considered as a method of reducing $\mathrm{S}$. sanguinis and S. mutans in saliva, which results in reducing the dental plaque. The purpose of this study was to evaluate the effects of NH on the growth of S. sanguinis and to compare its effects with that of Ciprofloxacin and Penicillin antibiotics in vitro. Materials and Methods: In this experimental study, the effect of NH against S. sanguinis was investigated according to the growth inhibition zone in the disk diffusion method by measuring the inhibition zone around the disks which were dipped in the case and control groups. The agar well diffusion method was used as well. The results were analyzed using analysis of variance (ANOVA). In case of the presence of a significant difference, post-hoc Scheffe test was used as a supplementary test.

Results: In the disk diffusion method, the average inhibition zone was $21 \mathrm{~mm}$ for Ciprofloxacin, $10.35 \mathrm{~mm}$ for Penicillin, and $17.77 \mathrm{~mm}$ for $\mathrm{NH}(\mathrm{P}<0.001)$. In the agar well diffusion method, the average inhibition zone was $17.68 \mathrm{~mm}$ for Ciprofloxacin, $15.83 \mathrm{~mm}$ for $\mathrm{NH}$, and $8.06 \mathrm{~mm}$ for Penicillin $(\mathrm{P}<0.01)$.

Conclusion: It seems that $\mathrm{NH}$ is effective against $\mathrm{S}$. sanguinis in vitro. Nevertheless, its inhibition zone in the agar well diffusion and the disk diffusion methods is smaller than that formed by Ciprofloxacin and larger than that formed by Penicillin.

Keywords: Anti-Bacterial Agents, Honey, Streptococcus sanguinis

\section{J Res Dent Maxillofac Sci 2019;4(1):1-8.}

DOI: 10.29252/jrdms.4.1.1

\section{Introduction:}

Dental caries and periodontal diseases are considered as common diseases in the oral environment, which are caused by the accumulation of oral microorganisms in saliva on dental surfaces due to inappropriate diet and habits.
An important part of the dental plaque is comprised of oral Streptococci. One of these microorganisms is Streptococcus sanguinis ( $\mathrm{S}$. sanguinis) which plays an important role in the formation of dental caries. ${ }^{(1-3)}$ S. sanguinis is considered as a primary colonized microorganism in 
dental plaque. ${ }^{(4,5)}$ Also, the numbers of S. sanguinis are significantly higher in individuals with high rates of decay than in individuals with no decay. ${ }^{(6)}$ Nowadays, different methods have been proposed to prevent dental caries by reducing $\mathrm{S}$. sanguinis and Streptococcus mutans (S. mutans), including the use of antibacterial agents such as chlorhexidine (CHX), causing interference with the metabolism of microbes by Xylitol, blocking the adhesion of these microorganisms to dental surfaces by receptor analogs, and use of vaccines for caries. $^{(7)}$ Non-prevention of dental caries, in addition to imposing high costs, leads to loss of teeth due to decay, aesthetic defects, decreased chewing ability, pain, oral and digestive disorders, nutritional deficiencies, etc. ${ }^{(8)}$ Extensive research is currently in progress on the use of natural honey $(\mathrm{NH})$ in the diet as a method for reducing $\mathrm{S}$. sanguinis and $\mathrm{S}$. mutans in saliva with subsequent reduction of dental plaque as the initiator of the decay process. According to the results presented in traditional medicine, honey has the power to decrease these microorganisms. ${ }^{(9-14)}$ Due to the shortcomings of previous research methods in this field, which have been carried out in vivo, as before-after interventions or experimentally on certain strains of bacteria, ${ }^{(15-20)}$ this study aimed to examine the effects of $\mathrm{NH}$ on the growth rate of $\mathrm{S}$. sanguinis at the Dental Faculty of Islamic Azad University of Medical Sciences, Tehran, Iran.

\section{Materials and Methods}

This in-vitro experimental study was performed during October 2016-August 2017. The study population included S. sanguinis strains (PTCC 1449).

Penicillin and Ciprofloxacin antibiotics were prepared according to the table developed by the Clinical \& Laboratory Standards Institute (CLSI). In this study, $\mathrm{NH}$ at a concentration of $1200 \mathrm{ppm}$ (parts-per-million) was prepared from the southern province of Fars (Qir and Karzin) in late October. ${ }^{(21)}$

In the present study, $26 \mathrm{~S}$. sanguinis (PTCC 1449) samples, including 8 samples in the Ciprofloxacin group, 8 samples in the Penicillin group, and 8 samples in the NH group were cultured in Mueller-Hinton Broth (MHB) culture medium. Two samples were considered as negative controls, on which, $\mathrm{NH}$ was placed using a blank disk to be compared to Penicillin and Ciprofloxacin. After 24 hours at $37^{\circ} \mathrm{C}$, the inhibition zones of Penicillin, Ciprofloxacin, and NH were measured and calculated. Also, the minimum inhibitory concentration (MIC) of bacterial growth and the minimum bactericidal concentration (MBC) were determined for the above. ${ }^{(22)}$

\section{Sampling method:}

The convenience sampling method was used to select samples from S. sanguinis strains (PTCC 1449).

At first, a live culture sample of S. sanguinis bacteria (PTCC 1449), prepared by the Iranian Research Organization for Science and Technology (IROST), was cultivated for 24 hours at $37^{\circ} \mathrm{C}$ in a blood agar medium and in anaerobic conditions in a GasPak jar for recovery and strengthening in order to reach its maximum development. After 24 hours, bacterial colonies were removed, and based on $0.5 \mathrm{McF}$ arland turbidity, they were dissolved in 2 cc of physiologic serum, which confirms that the number of bacteria present in the serum is equivalent to $1.5 \times 10^{8}$ colony-forming units $(\mathrm{CFU}) /$ milliliter $(\mathrm{ml})$. Then, the bacteria were removed from the solution using sterile swabs and were cultivated in a blood agar medium. Next, a blank disc dipped in NH was placed on the culture medium. ${ }^{(23)}$

Also, Ciprofloxacin and Penicillin disks were used for the control group. Blank disks were used as negative controls. After placing the discs, the plates were incubated at $37^{\circ} \mathrm{C}$, and after 24 hours, the inhibition zone around the discs was measured in millimeter $(\mathrm{mm})$ using a metallic ruler. Another method to perform this test is to create a well in the plate. Wells with a diameter of $7 \mathrm{~mm}$ were created on a solid culture media, and $1.1 \mathrm{ml}$ of $\mathrm{NH}$ was poured into the wells. Also, $1.1 \mathrm{ml}$ of each of Ciprofloxacin and Penicillin antibiotics (by calculating the potency of the antibiotic) were poured into the wells using a standard loop. 
The plates were incubated at $37^{\circ} \mathrm{C}$ for 24 hours. Next, the inhibition zone ( $\mathrm{mm}$ ) was measured using the metallic ruler.

Determining the sensitivity of the microbe to $\mathrm{NH}$ using the agar well diffusion method: The experiment was performed to investigate the effect of $\mathrm{NH}$, to test the sensitivity of S. sanguinis using the agar well diffusion method, and to evaluate the diameter of the inhibition zone.

S. sanguinis was cultured on a specific solid culture medium. Wells were created with a diameter of $7 \mathrm{~mm}$ apart on the solid culture medium, and $1.1 \mathrm{ml}$ of $\mathrm{NH}$ was poured into the wells using a standard loop; Penicillin and Ciprofloxacin antibiotics were prepared based on the concentration potency, and $1.1 \mathrm{ml}$ of each antibiotic was poured into each well. The plates were exposed to microaerophilic conditions in the presence of carbon dioxide $(\mathrm{CO} 2)$ and were incubated at $37^{\circ} \mathrm{C}$ for 48 hours. ${ }^{(21,24)}$

Also, in order to determine the antibacterial activity in this study, the dilution method was used to determine the MIC and MBC. ${ }^{(25)}$

\section{The dilution method:}

The MIC refers to the antibacterial capacity of a substance or its ability to prevent the proliferation of microorganisms. In other words, the lowest concentration of a substance that can prevent the growth of microorganisms is called its MIC. Both the macrodilution broth and the microdilution broth techniques, recommended by the CLSI, were used to evaluate the MIC in the MHB culture medium. ${ }^{(7)}$

\section{The macrodilution broth technique:}

At first, $0.5 \mathrm{ml}$ of MHB culture medium was added to 8 test tubes and sterilized in an autoclave. Then, in order to prepare various dilutions, $0.5 \mathrm{ml}$ of $\mathrm{NH}$ was added to a tube containing $0.5 \mathrm{ml}$ of MHB medium, and after several times of mixing and sampling, $0.5 \mathrm{ml}$ of the contents of the tube was taken and added to the first of the 8 tubes, and thus, we diluted the contents of the first tube (1:2). Then, $0.5 \mathrm{ml}$ from the first tube was transferred to the second tube to obtain a dilution of $1: 4$. This process was continued up to the eighth tube. Then, 0.5 $\mathrm{ml}$ of the contents of the eighth tube was discarded, and the contents were diluted to a concentration of $1: 256$.
Also, a test tube was used as a negative control, containing $0.5 \mathrm{ml}$ of MHB medium and $0.5 \mathrm{ml}$ of $\mathrm{NH}$. Then, $0.5 \mathrm{ml}$ of the microfluidics suspension at a concentration of $0.5 \mathrm{McF}$ arland was added to each of the tubes, except for the tube considered as the negative control. The tubes were incubated for 24 hours. This was done for Ciprofloxacin and Penicillin antibiotics as well. In order to ensure the accuracy of the test, the process was repeated 6 times under aseptic, protected conditions.

\section{The microdilution broth technique:}

Microdilution with the microplate method was another method used in this experiment. First, 100 $\lambda$ of MHB medium were poured into each well. Then, $100 \lambda$ of MHB and $100 \lambda$ of NH were mixed in a tube. After several samplings, $100 \lambda$ were taken from this tube and added to the first well. Then, 100 $\lambda$ from the first well was transferred to the second well; this was continued to the eighth well to obtain the proper dilution. Then, $0.5 \mathrm{McF}$ arland of the bacteria was diluted at the ratio of 1:20 (50 $\lambda$ of 0.5 McFarland of the bacteria $+950 \lambda$ of physiologic serum), and $10 \lambda$ from this concentration was added per well. This was done for Ciprofloxacin and Penicillin antibiotics as well. Then, the microplates were incubated for 24 hours. MIC results are measurable with unaided eye after the test; therefore, we reported the MIC in the last tube (macrodilution broth) with no turbidity. ${ }^{(23)}$ To ensure the accuracy of the results, we also performed MBC testing to precisely determine the MIC.

In order to determine the $\mathrm{MBC}$, first, blood agar culture medium was placed in a plate, and then, the contents of the tube that was reported as the MIC and the two following tubes in which no turbidity was observed were cultured in a blood agar medium and incubated for 24 hours.

Table 1. The diameter $(\mathrm{mm})$ of inhibition zones according to the agar well diffusion method in the studied groups

\begin{tabular}{ccc} 
Groups & $\begin{array}{c}\text { Diameter } \\
(\mathrm{mm})\end{array}$ & $\mathrm{CV}$ \\
\hline Natural & 10 \\
Honey & $1.89 \pm 17.77$ & 10 \\
\hline Ciprofloxacin & $2.20 \pm 21.00$ & 10 \\
\hline Penicillin & $1.74 \pm 10.35$ & 16 \\
\hline
\end{tabular}

$\mathrm{CV}=$ Coefficient of Variation 
A portion of the plate with no bacterial growth was considered as the MBC. This was done for Ciprofloxacin and Penicillin antibiotics as well. ${ }^{(25)}$

Analysis of variance (ANOVA) was performed for the three groups; in case of the presence of a significant difference, post-hoc Scheffe test was used as a supplementary test.

\section{Results:}

This study was carried out on 26 samples including 8 samples in the Ciprofloxacin group, 8 samples in the Penicillin group, and 8 samples in the $\mathrm{NH}$ group. In this study, two negative controls were included, in which, no zone of inhibition was observed around the disk and the well.

The diameters of the inhibition zones in the Ciprofloxacin, $\mathrm{NH}$, and Penicillin wells are presented in Table 1 which shows that the mean \pm standard deviation (SD) diameter of the inhibition zone was $21 \pm 2.20 \mathrm{~mm}$ for Ciprofloxacin, $10.35 \pm 1.74 \mathrm{~mm}$ for Penicillin, and $17.77 \pm 1.89 \mathrm{~mm}$ for $\mathrm{NH}$; the diameter in the NH group was $7.42 \mathrm{~mm}$ larger than that in the Penicillin group. Also, in the Ciprofloxacin group, the diameter was $10.65 \mathrm{~mm}$ larger than that in the NH group; accordingly, the maximum and the minimum diameters of inhibition zones were detected in the Ciprofloxacin group and in the Penicillin group, respectively. ANOVA showed a statistically significant difference in the inhibition zones among the three groups $(\mathrm{P}<0.01)$. According to the results of post-hoc Scheffe test, there were significant differences between the NH group and the Penicillin group, between the Ciprofloxacin group and the NH group, and also between the Ciprofloxacin group and the Penicillin group $(\mathrm{P}<0.01)$. In addition, the highest coefficients of variation $(\mathrm{CV})$ of $\mathrm{NH}$ and Ciprofloxacin were equal to 10 . However, the CV for Penicillin was 16.

The diameters of the inhibition zones in the disk diffusion method according to the groups are presented in Table 2. This table demonstrates that the diameter of the inhibition zone is $17.68 \mathrm{~mm}$ in the Ciprofloxacin group, $15.83 \mathrm{~mm}$ in the $\mathrm{NH}$ group, and $8.06 \mathrm{~mm}$ in the Penicillin group; the diameter is $77.7 \%$ larger in the NH group than in the Penicillin group, and $1.85 \mathrm{~mm}$ larger in the Ciprofloxacin group compared to the NH group. ANOVA showed that this difference was statistically significant $(\mathrm{P}<0.001)$. The $\mathrm{CV}$ was 7 in the Ciprofloxacin group, 9 in the $\mathrm{NH}$ group, and 17 in the Penicillin group.

Table 2. The diameter $(\mathrm{mm})$ of inhibition zones according to the disk diffusion method in the studied groups

\begin{tabular}{ccc}
\hline Groups & $\begin{array}{c}\text { Diameter } \\
(\mathrm{mm})\end{array}$ & $\mathrm{CV}$ \\
\hline $\begin{array}{l}\text { Natural } \\
\text { Honey }\end{array}$ & $1.49 \pm 15.83$ & 9 \\
\hline Ciprofloxacin & $1.28 \pm 17.68$ & 7 \\
\hline Penicillin & $1.42 \pm 8.06$ & 17 \\
\hline
\end{tabular}

$\mathrm{CV}=$ Coefficient of Variation

In this study, the MIC and the MBC for $\mathrm{NH}$ and the two antibiotics were measured, and the results are presented in Table 3.

Table 3. The minimum inhibitory concentration (MIC) and the minimum bactericidal concentration (MBC) in the studied groups

\begin{tabular}{cccc} 
& With & Without & Total \\
& eyeglasses & eyeglasses & \\
\hline Good & $16(1.57 \%)$ & $0(0 \%)$ & $16(1.57 \%)$ \\
\hline Fair & $10(7.35 \%)$ & $1(6.3 \%)$ & $11(3.39 \%)$ \\
\hline Poor & $1(6.3 \%)$ & $0(0 \%)$ & $1(6.3 \%)$ \\
\hline Total & $27(4.96 \%)$ & $1(6.3 \%)$ & $28(100 \%)$ \\
\hline
\end{tabular}


According to the results of this study, the maximum MIC was for the Penicillin group (0.057 $\mathrm{mg} / \mathrm{ml}$ ), and the minimum MIC was for the Ciprofloxacin group $(0.003 \mathrm{mg} / \mathrm{ml})$; the results presented an insignificant difference among the three groups $(\mathrm{P}=0.4)$.

The maximum MBC $(0.25 \mathrm{mg} / \mathrm{ml})$ was observed in the Penicillin group, and the minimum MBC $(0.031 \mathrm{mg} / \mathrm{ml})$ was detected in the Ciprofloxacin group; the test showed a statistically significant difference regarding the MBC in the three groups, and post-hoc Scheffe test demonstrated a difference in pairwise comparisons.

According to the results of the statistical analyses, Ciprofloxacin had a superior inhibitory effect on the bacteria. The effect of $\mathrm{NH}$ was superior to that of Penicillin; therefore, $\mathrm{NH}$ can be considered an inhibitor and an appropriate bactericidal agent against $\mathrm{S}$. sanguinis. The substance that shows inhibitory effects in lower dilutions is considered as a more proper option.

\section{Discussion:}

According to the results of this study, $\mathrm{NH}$ has a significant effect on the diameter of the inhibition zone against $\mathrm{S}$. sanguinis in vitro. However, the diameter of the inhibition zone of S. sanguinis in the agar well diffusion method and in the disk diffusion method was less than that of Ciprofloxacin, but it was more efficient against the growth of the bacteria. Also, in this study, the MIC and the $\mathrm{MBC}$ were measured for $\mathrm{NH}$ and the two antibiotics. According to the results, $\mathrm{NH}$ is superior to Penicillin regarding its effect on the above microorganisms. In terms of the $\mathrm{MBC}$ and the MIC, Ciprofloxacin was the most effective agent. However, it should be noted that NH, which has no side effects, can be a good alternative for the treatment and the prevention of the colonization of these bacteria.

In 2017, Mathai et al evaluated the effect of lemon, ginger, garlic, and honey extracts on S. mutans using the well diffusion method; ${ }^{(11)}$ garlic showed the greatest antimicrobial effect against S. mutans. This study confirms the antimicrobial effects of $\mathrm{NH}$ although the type of the studied bacteria is different from that studied in our research.

In 2016, Khozeymeh et al investigated the antibacterial effect of Astragalus honey on three
Lactobacilli species involved in dental caries by serial dilutions and the disk diffusion method and showed that Astragalus honey is effective on Lactobacilli and can be used as a natural antibacterial agent. ${ }^{(23)}$ The results of this study are consistent with our study and confirm the antimicrobial properties of $\mathrm{NH}$, although the type of studied honey and the bacteria are different from those in our study. S. sanguinis is considered as a common pathogenic oral bacterium that was examined in this study. We compared the antimicrobial properties of $\mathrm{NH}$ with two types of antibiotics, which is one of the strengths of the present study.

Ghotaslou et al investigated the antibacterial effect of honey in Azerbaijan on the Pseudomonas aeruginosa biofilm by the MIC and MBC methods in 2016 and showed that honey has an antimicrobial effect on planktonic Pseudomonas aeruginosa, and it was concluded that honey has significant anti-biofilm activity. (25) Their results are consistent with our study, confirming the antibacterial properties of honey. However, the type of honey and the tested bacterial species are different from those in our study. In addition, in the present study, the antimicrobial effect of honey was compared with that of two different antibiotics, allowing for the qualitative examination of the antimicrobial properties of $\mathrm{NH}$, and this is considered as the strength of our study. In 2014, Atwa et al examined the effects of honey on the prevention of gingival inflammation and dental caries in patients undergoing orthodontic treatment. ${ }^{(26)}$ They measured plaque $\mathrm{pH}$ after eating honey with control solutions $(10 \%$ sucrose or $10 \%$ sorbitol) and determined the number of bacteria in the plaque. According to the results, honey had a significant positive effect on reducing bacteria such as S. mutans, Lactobacillus, and Porphyromonas gingivalis which are the causes of gingival inflammation and dental caries. ${ }^{(26)}$ The result of the cited study is consistent with that of our study, but the type of studied bacteria and their study method are different from those of our study. In that study, the bacteria were isolated from dental plaque, but in our study, a standard bacterial sample was prepared from the reference center.

In 2013, Khosravi-Darani et al assessed the anticonvulsant and antibacterial effects of three 
types of Iranian honey with alcoholic extracts of peppermint, thyme, ginger, and starch on Staphylococcus aureus, Pseudomonas aeruginosa, Escherichia coli (E. coli), and Candida albicans. ${ }^{(27)}$ They concluded that the addition of the above herbal extracts improves the antibacterial and antifungal properties of honey and they can be considered as an alternative method when there is resistance against antibiotics. ${ }^{(27)}$

Three types of honey and other herbal extracts were used in the mentioned study, and the bacterial species studied were also different from that of our study. Also, they showed the antifungal properties of honey, but the result is consistent with our study in terms of the confirmation of the antibacterial properties of honey.

In 2011, Kwakman et al examined the antibacterial activity of two different honey types on E. coli, Bacillus subtilis, and Pseudomonas aeruginosa. ${ }^{(28)}$ They concluded that the antibacterial property of $\mathrm{NH}$ is related to different bee defensins, methylglyoxal (MGO), and hydrogen peroxide (H2O2) which are present at different levels in different types of honey. This means that all types of $\mathrm{NH}$ are bactericidal agents but they have different powers. ${ }^{(28)}$ Of course, the bacteria used in the mentioned study were different from those studied in our research. In 2012, Nassar et al investigated the effect of honey on S. mutans growth and biofilm formation. ${ }^{(29)}$ The results demonstrated the potential antibacterial properties of $\mathrm{NH}$ and suggested that the antimicrobial property of $\mathrm{NH}$ is not solely due to its high sugar content. ${ }^{(29,30)}$

The result of the cited study is also consistent with that of the present study but the studied bacterial species was different.

Shoae Hassani et al carried out similar work on determining the MIC; they investigated the effect of ethyl acetate extract of beeswax on the colonization of S. mutans in 2009. ${ }^{(31)}$ The difference between their study and the present study is that they used beeswax but we used pure honey, and also, the bacteria studied by them were different from those studied here. Furthermore, we determined the inhibition zone in addition to the MIC and MBC.

In 2015, Jain et al investigated the effects of honey, CHX mouthwash, and Xylitol chewing gum plus $\mathrm{CHX}$ mouthwash in vivo on the plaque surface; they concluded that the honey group and the CHX plus Xylitol group were more effective in decreasing the plaques. $^{(32)}$

The cited study describes the reduction in dental plaque following honey use, which is inconsistent with our study that showed a direct reduction in the number of cariogenic microorganisms, but since there are different cariogenic microorganisms in dental plaque, their study also confirms the antimicrobial properties of honey in line with our study.

The present study was carried out in vitro, and the results may not be generalized to in-vivo conditions. On the other hand, the S. sanguinis strain used in the present research was a standard laboratory sample and it was impossible to collect $S$. sanguinis from the patients' mouths. If we could use different types of honey at different concentrations in the present study, the results would be affected; nevertheless, we tried to avoid bias in this study.

One of the advantages of the present study is that the antimicrobial activity of honey was investigated at the Department of Microbiology of Shahid Beheshti University of Medical Sciences based on the CLSI protocol. In this study, the disk diffusion, MIC, and MBC methods were used in addition to the well diffusion method, and each stage was repeated six times by a single operator on different days; the frequency of testing was high.

\section{Conclusion:}

The results of the present study indicated that NH is effective against S. sanguinis in vitro. Nevertheless, its zone of inhibition in the agar well diffusion and the disk diffusion methods was smaller than that formed by Ciprofloxacin and greater than that formed by Penicillin.

\section{Acknowledgments:}

Special thanks to our teachers and to the laboratory staff of Shahid Beheshti University of Medical Sciences for their cooperation. This paper was written based on thesis no. 25501. 


\section{Conflict of interests:}

The authors declare no potential conflict of interests with respect to the research, authorship, and/or publication of this article.

This research received no specific grant from any funding agency in the public, commercial, or not-for-profit sector.

\section{References:}

1-He XS, Shi WY. Oral microbiology: past, present and future. Int J Oral Sci. 2009 Jun;1(2):47-58.

2-Marsh PD. Microbiology of dental plaque biofilms and their role in oral health and caries. Dent Clin North Am. 2010 Jul;54(3):44154.

3-Aas JA, Griffen AL, Dardis SR, Lee AM, Olsen I, Dewhirst FE, et al. Bacteria of dental caries in primary and permanent teeth in children and young adults. J Clin Microbiol. 2008 Apr;46(4):1407-17.

4-Yim N, Ha do T, Trung TN, Kim JP, Lee $\mathrm{S}, \mathrm{Na} \mathrm{M}$, et al. The antimicrobial activity of compounds from the leaf and stem of Vitis amurensis against two oral pathogens. Bioorg Med Chem Lett. 2010 Feb 1;20(3):1165-8. 5-Doyle RJ, Nesbitt WE, Grant Taylor K. On the mechanism of adherence of Streptococcus sanguis to hydroxylapatite. FEMS Microbiol Lett. 1982 Sep;15(1):1-5.

6-Giacaman RA, Torres S, Gomez Y, MunozSandoval C, Kreth J. Correlation of Streptococcus mutans and Streptococcus sanguinis colonization and ex vivo hydrogen peroxide production in carious lesion-free and high caries adults. Arch Oral Biol. 2015 Jan;60(1):154-9.

7-Rotilie CA, Fass RJ, Prior RB, Perkins RL. Microdilution technique for antimicrobial susceptibility testing of anaerobic bacteria. Antimicrob Agents Chemother. 1975 Mar;7(3):311-15.

8-Featherstone JD. Caries prevention and reversal based on the caries balance. Pediatr Dent. 2006 Mar 1;28(2):128-32.

9-Abd El-Malek FF, Yousef AS, El-Assar SA. Hydrogel film loaded with new formula from manuka honey for treatment of chronic wound infections. J Glob Antimicrob Resist. 2017 Dec;11:171-6.
10-Singhal R, Siddibhavi M, Sankeshwari R, Patil P, Jalihal S, Ankola A. Effectiveness of three mouthwashes - Manuka honey, Raw honey, and Chlorhexidine on plaque and gingival scores of 12-15-year-old school children: A randomized controlled field trial. J Indian Soc Periodontol. 2018 Jan-Feb;22(1):34-9.

11-Mathai K, Anand S, Aravind A, Dinatius P, Krishnan AV, Mathai M. Antimicrobial Effect of Ginger, Garlic, Honey, and Lemon Extracts on Streptococcus mutans. J Contemp Dent Pract. 2017 Nov 1;18(11):1004-8.

12-Molan PC. The role of honey in the management of wounds. J Wound Care. 1999 Sep;8(8):415-8.

13-Nejabat M, Astaneh A, Eghtedari M, Mosallaei M, Ashraf MJ, Mehrabani D. Effect of honey in Pseudomonas aeruginosa induced stromal keratitis in rabbits. J Appl Animal Res. 2009 Jun 1;35(2):101-4.

14-Schmidlin PR, English H, Duncan W, Belibasakis GN, Thurnheer T. Antibacterial potential of Manuka honey against three oral bacteria in vitro. Swiss Dent J. 2014;124(9):922-4.

15-Leandro LF, Mendes CA, Casemiro LA, Vinholis AH, Cunha WR, de Almeida R, et al. Antimicrobial activity of apitoxin, melittin and phospholipase A2 of honey bee (Apis mellifera) venom against oral pathogens. An Acad Bras Cienc. 2015 Mar;87(1):147-55.

16-Andualem B. Combined antibacterial activity of stingless bee (Apis mellipodae) honey and garlic (Allium sativum) extracts against standard and clinical pathogenic bacteria. Asian Pac J Trop Biomed. 2013 Sep;3(9):725-31.

17-Molan PC. The antibacterial activity of honey: 1 . The nature of the antibacterial activity. Bee World. 1992 Jan 1;73(1):5-28.

18-Bauer L, Kohlich A, Hirschwehr R, Siemanna U, Ebner H, Scheiner O, et al. Food allergy to honey: Pollen or bee products? Characterization of allergenic proteins in honey by means of immunoblotting. J Allergy Clin Immunol. 1996 Jan 1;97(1):65-73.

19- Duailibe SA, Gonçalves AG, Ahid FJ. Effect of a propolis extract on Streptococcus mutans counts in vivo. J Appl Oral Sci. 2007 Oct;15(5):420-3.

20-Sela MO, Maroz D, Gedalia I. Streptococcus Mutans in saliva of normal subjects and neck and head irradiated cancer subject after consumption 
of honey. J Oral Rehabil. 2000;27:269-270.

21-Performance Standards for Antimicrobial Susceptibility Testing. 28th ed. CLSI supplement M100. Wayne, PA: Clinical and Laboratory Standards Institute; 2018.

22-Vahabi S, Najafi E, Alizadeh S. In vitro antimicrobial effects of some herbal essences against oral pathogens. J Med Plants Res. 2011;5(19):4870-8.

23-Khozeymeh F, Golestannejad Z, Aayan A. Antibacterial Effect of Astrangalus Honey on Cariogenic Lactobacillus Strains. J Isfahan Dent Sch. 2016;11(6):45-62.

24-Allen KL, Molan PC, Reid GM. A survey of the antibacterial activity of some New Zealand honeys. J Pharm Pharmacol. 1991 Dec;43(12):817-822.

25-Ghotaslou R, Saghati H, Dehnad A, Salahi Eshlaghi B. Antibacterial Effect of Azerbaijan Honey on Pseudomonas aeroginosa Biofilm. Iran J Med Microbiol. 2016;9(4):40-6.

26-Atwa AD, AbuShahba RY, Mostafa M, Hashem MI. Effect of honey in preventing gingivitis and dental caries in patients undergoing orthodontic treatment. Saudi Dent J. 2014 Jul;26(3):108-14.

27-Khosravi-Darani K, Khaksar R, Esmaeili S, Seyed-Reihani F, Zoghi A, Shahbazizadeh S. Antifungal and Anti-bacterial Synergistic Effects of Mixture of Honey and Herbal Extracts. ZJRMS. 2013;15(8):30-3.

28-Kwakman PH, Te Velde AA, de Boer L, Vandenbroucke-Grauls CM, Zaat SA. Two major medicinal honeys have different mechanisms of bactericidal activity. PloS One. 2011 Mar 4;6(3):e17709.

29-Nassar HM, Li M, Gregory RL. Effect of honey on Streptococcus mutans growth and biofilm formation. Appl Environ Microbiol. 2012 Jan;78(2):536-40.

30- Moezzi ghadim N, Taghibakhsh M, Godarzi H, Liravinezhad hoseini N, Alirezaei S. Evaluation of the Effect of Four Herbal Extracts on Growth of Streptococcus mutans and Lactobacillus. J Res Dentomaxillofac Sci. 2018;3(2):7-13. 31-Shoae Hassani AR, Hamdi K, Ghaemi A. In vitro reduction in colonization of Streptococcus mutans by honey beeswax ethyl acetate extract. AMUJ. 2009 Winter;11(4):87-95.

32-Jain A, Bhaskar DJ, Gupta D, Agali C, Gupta

V, Gupta RK, et al. Comparative evaluation of honey, chlorhexidine gluconate $(0.2 \%)$ and combination of xylitol and chlorhexidine mouthwash $(0.2 \%)$ on the clinical level of dental plaque: A 30 days randomized control trial. Perspect Clin Res. 2015 Jan-Mar;6(1):53-7.

Please cite this paper as:

Alaee A, Azizi A, valaei N, Taheri S, Ravanshadi F, Fattahi M. In-Vitro Effect of Natural Honey on the Growth of Streptococcus sanguinis . J Res Dentomaxillofac Sci. 2019; 4 (1) :1-8 